\title{
Towards Proactive Web Service Adaptation
}

\author{
Ahmed Moustafa and Minjie Zhang \\ School of Computer Science and Software Engineering, \\ University of Wollongong, Gwnneville, NSW 2500, Australia \\ \{aase995,minjie\}@uowmail.edu.au \\ http://www.uow.edu.au
}

\begin{abstract}
With the rapid development of web service technology, next generations of web service applications need to be able to predict problems, such as potential degradation scenarios, future erroneous behaviors and deviations from expected behaviors, and move towards resolving those problems not just reactively, but even proactively, i.e., before the problems occur. Service oriented applications are thus driven by the requirements that bring the concepts of decentralization, dynamism, adaptation, and automation to an extreme. In this paper, an approach is proposed that depends mainly on the concept of proactive adaptation by the use of reinforcement learning to achieve an autonomous dynamic behavior of web service composition considering potential degradation and emergence in $Q o S$ values. Experimental results show the effectiveness of the proposed approach in dynamic web service composition environments.
\end{abstract}

Keywords: Web services, dynamic composition, proactive adaptation.

\section{Introduction}

Research in web service composition has drawn a great deal of attention in recent years. The service composition process can be distinguished as fulfilling user requirements using available web services repositories. Those requirements can be functional or non-functional. While the former is in relation to targeting the overall outcome concerning the the underway business process. The latter pertains to the quality of the composition as a whole in terms of availability, reliability, cost and response time.

Although many standards are being adopted in this domain, lack of QoS support is a long standing main challenge and an obvious drawback of current composition engines. Hence, efficient web services applications need to be managed not only from a functional perspective but also non-functional qualities should be incorporated.

The importance of service adaptation has generated a cross domain interest spanning a number of research fields. Web service adaptation is of adjusting web services to changing user requirements, QoS degradations and other prospective run time environment changes. Changes in run time environment can be in the form of QoS change, functional requirements change or environment change. 
Any service composition approach should take into consideration the above forms of run time environment changes and work towards getting through those limitations. This paper mainly targets overcoming the consequent limitation of QoS change with an aim to provide an adaptive approach for service composition that can work in a proactive mode.

Initial attempts of $Q o S$ monitoring and adaptation for composite Web services focused on AI planing techniques, Genetic Algorithms and Formal Methods 121. Though those methods still lack proactivity, they assume the corrective adaptation behavior to happen after a problem occurs which is not the case for many real world applications. For example, critical real time systems need to accommodate predictive proactive activity that is able to forecast the erroneous behavior and possible degradations in $Q \circ S$ before it takes place.

In this paper, we propose an approach to integrate both proactive and adaptive behavior into web service composition. The proposed approach has three contributions in terms of its abilities of prediction, incorporation and learning of web service composition attitude. Those abilities can be briefed as: the ability to predict possible downfall of the composite services quality during run time, the ability to incorporate single services and composite workflows dynamically into the running composition and the ability to learn QoS attributes of the undergoing services dynamically at run time. Having those qualities, the proposed approach outperforms other existing approaches of service composition and optimization that assume an abstract static composition environment which is not the case for real world applications.

The rest of this paper is organized as follows. Section 2 communicates a detailed methodology and an approach description. Experiments are conducted and results are assessed in Section 3. Section 4 gives a brief review of related work and discussions. Finally, conclusions are drawn in Section 5.

\section{Methodology}

In this section, a multi-phase approach is proposed to address the problem of web service adaptation in a composite dynamic environment. The main approach consists of three phases as follows:

\section{Phase One: Modeling the Service Composition Process}

Having a user request, the approach matches the user functional requirements and generates an abstract logical workflow that is subsequently adopted by selecting the best available QoS.

\section{Phase Two: Web Service Log Analysis}

Once deployed, the proposed approach dynamically monitors the composition via analyzing the historical data in the web service execution log.

\section{Phase Three: Proactive Adaptation}

Recognizing the dynamic environment, the proposed approach adaptively learns the best execution policy and proactively takes corrective actions to keep the workflow running in a dynamic and smooth manner. 
The three phases are introduced in detail in the following subsections, respectively.

\subsection{Phase 1: Modeling the Service Composition Process}

Markov Decision Process (MDP) is an AI method to model sequential decision processes and has been also used in different applications. In this phase, we employ MDP concept to model the process of service composition as it can enable to model service composition in dynamic environments as a series of steps showing stochastic nature [57]. We employed MDP concept in our approach to model the service composition process.

In general, a web service can be described in terms of service ID and quality of service $Q o S$. A web service can be formally defined by Definition 1.

Definition 1 (Web Service). A $W e b$ Service $W S$ is defined as a tuple $W S=<$ $I D, Q o S>$, where $I D$ is the identifier of the web service, $Q o S$ is the quality of the service represented by a $n$-tuple $\left\langle Q_{1} ; Q_{2} ; \ldots ; Q_{n}>\right.$, where each $Q_{i}$ denotes a $Q o S$ attribute of $W S$.

A general Markov Decision Process (MDP) is defined as follows [577.

Definition 2 (Markov Decision Process (MDP)). A MDP is defined as a 4-tuple $M D P=<S, A(), P,. R>$, where

$-S$ is a finite set of states of the world.

- $A(s)$ is a finite set of actions depending on the current state $s \in S$.

$-P$ is a probability value, i.e., when an action $a \in A$ is performed, the world makes a probabilistic transition from its current state $s$ to a resulting state $s^{\prime}$ according to a probability distribution $P\left(s^{\prime} \mid s, a\right)$

$-R$ is a reward function. Similarly, when action $a$ is performed the world makes its transition from $s$ to $s^{\prime}$, the composition receives a real-valued reward $r$, whose expected value is $r=R\left(s^{\prime} \mid s, a\right)$.

A MDP involves multiple actions and paths to choose. By using this feature to model service compositions, it can enable us to integrate multiple alternative workflows and services into a single composition by simply replacing the actions in a MDP with single web services or composite workflows. Based on this consideration, we develop a MDP-based service composition model in this phase, which is formally defined by Definition 3 .

Definition 3 (A MDP-Based Web Service Composition (MDP-WSC)). A MDP-Based Web Service Composition is defined as a 6 -tuple, MDP-WSC $=<$ $S, s_{0}, S_{r}, A(), P,. R>$, where

$-S$ is a finite set of states of the world;

$-s_{0} \in S$ is the initial state and any execution of the the service composition usually starts from this state;

$-S_{r} \subset S$ is the set of terminal states. Upon arriving at one of those states, an execution of the service composition terminates. 
- $A(s)$ is the set of web services that can be executed in state $s \in S$, a service $W S$ belongs to $A(s)$, only if the precondition $W S^{P}$ is satisfied by $s$.

- $P$ is the probability when a web service $W S \in A(s)$ is invoked when the world makes a transition from its current state $s$ to a resulting state $s^{\prime}$, where the effect of $W S$ is satisfied. For each $s$, the transition occurs with a probability $P\left(s^{\prime} \mid s, W S\right)$.

- $R$ is a reward function when a web service $W S \in A(s)$ is invoked, the environment makes a transition from $s$ to $s^{\prime}$, and the service consumer receives an immediate reward $r$, whose expected value is $R\left(s^{\prime} \mid s, W S\right)$.

the above MDP-WSC model is expressive enough to describe the control flows of general service compositions. In particular, the proposed MDP-WSC model can express a super service composition which in turn is composed of multiple smaller compositions. This feature enables to incorporate single services as well as alternative composite workflows to the deployed service composition dynamically.

\subsection{Phase 2: Web Service Log Analysis}

In software applications, logging refers to the recording of activities [156]. Throughout the service composition course, logging can be conducted at three different levels, which are an operational level, an interaction level and a workflow level. In this research, logging at the workflow level is employed to record for the behavior of the whole workflow. Execution logs accounting for the transactions of web services that are part of an ongoing workflow are collected from various web servers. Those logs are then merged into one coherent file.

Table 1 shows a sample of log file data auditing for $Q o S$ values on a transaction by transaction basis.

A service $W S$ is represented by the vector $W S=<I D, Q \circ S>$ (refer to Definition 1), where $I D$ is the identifier of the web service and $Q o S$ is an $n$-tuple < $Q_{1} ; Q_{2} ; \ldots ; Q_{n}>$, which represents the case of multiple attributed $Q o S$. For every transaction $t \in W S$, an aggregated value for $Q_{o} S$ attributes is calculated as follows:

$$
G(t)=\sum w \times \frac{Q_{i}^{t}-Q_{i}^{\min }}{Q_{i}^{\text {max }}-Q_{i}^{\text {min }}}
$$

where $Q_{i}^{t}$ represents the observed value of the $i t h$ attribute of a transaction t. $Q_{i}^{\max }$ and $Q_{i}^{\min }$ represent the maximum and minimum values of $Q_{i}$ for all

Table 1. Composition Transactions Log

\begin{tabular}{|c|c|c|c|c|c|}
\hline Service & Transaction & Time & Availability & Reliability & $G(t)$ \\
\hline \hline$W S_{1}$ & $t_{1}$ & $10: 40: 39$ & 0.92 & 0.4 & 0.73 \\
\hline$W S_{2}$ & $t_{1}$ & $10: 40: 44$ & 0.93 & 0.88 & 0.9 \\
\hline$W S_{1}$ & $t_{2}$ & $10: 40: 50$ & 0.94 & 0.77 & 0.83 \\
\hline$W S_{2}$ & $t_{2}$ & $10: 40: 54$ & 0.9 & 0.7 & 0.79 \\
\hline$W S_{2}$ & $t_{3}$ & $10: 41: 01$ & 0.8 & 0.92 & 0.83 \\
\hline$W S_{2}$ & $t_{4}$ & $10: 41: 02$ & 0.76 & .95 & 0.85 \\
\hline$W S_{1}$ & $t_{3}$ & $10: 41: 03$ & 0.96 & 0.99 & 0.97 \\
\hline$W S_{1}$ & $t_{4}$ & $10: 41: 09$ & 0.99 & 0.45 & 0.74 \\
\hline$W S_{2}$ & $t_{5}$ & $10: 40: 57$ & 0.99 & 0.91 & 0.95 \\
\hline
\end{tabular}


transactions recorded within the same service respectively. $w$ is the weighting factor of $Q_{i} . w$ is positive if users prefer $Q_{i}$ to be high, e.g. availability and reliability. Respectively, $w$ is negative if users prefer $Q_{i}$ to be low, e.g. service fee and response time. Calculated $G(t)$ values are then used as a basis for the rest of the approach.

\subsection{Phase 3: Proactive Adaptation}

In this phase, we use Q-learning [16] to address adaptation in web service environments. Q-learning is one of the methods in reinforcement learning. The reason for us to employ Q-learning in our adaptation phase is that (1) Q-learning is considered an effective strategy for learning optimal decisions during run time dynamic environments showing stochastic nature [1110] and (2) Q-learning does not require pre-training process which fits the situations of service oriented domains.

By using Q-learning inside our composition engine, it enables learning the optimal policy adaptively. Considering the MDP-WSC model introduced previously, the task of the service composition engine becomes distinguishing the optimal workflow that offers the best cumulative reward.

Definition 4 (Optimal Workflow). Let $W$ be the set of candidate workflows. Let $R_{i}^{w}$ be the reward associated with each Web service invocation $W S_{i}$ for some workflow $w$. Let $N$ be a fixed maximum number of invocations in each of the candidate workflows. The workflow $w^{*}$, that results in the maximum expected reward is called an optimal workflow.

This reward value is calculated using Q-learning [1110] as follows:

$$
Q(s, a) \leftarrow Q(s, a)+\alpha\left[r+\gamma \max _{a^{\prime}} Q\left(s^{\prime}, a^{\prime}\right)-Q(s, a)\right]
$$

where $s$ represents the state space for all the possible workflows the composition engine can go through to achieve a user's request, $a$ is the action vector representing the available web services, $r$ is the reward given by selecting particular service, $\alpha$ is a learning rate which controls convergence and $\gamma$ is the discount factor that reflects the learning policy. At the right side of Equation 2, $\alpha\left[r+\gamma \max _{a^{\prime}} Q\left(s^{\prime}, a^{\prime}\right)-Q(s, a)\right]$ represents the new immediate reward gained by selecting any service belongs to $a$ at any state belongs to $s$; whereas $Q(s, a)$ is the previously observed reward.

The learning process is conducted recursively through a sequence of episodes, in each episode the proposed approach starts from the initial state and selects a sequence of actions by following a learning policy 34 till reaches the goal state.

By incrementally updating the accumulative reward values gained each episode, the proposed approach can mix execution and learning at the same time. As the reward values are subject to change in a dynamic environment settings, the proposed approach can adapt to new rewards accordingly. 
This feature enables the proposed approach to promote real time adaptivity to dynamic service composition at run time.

Building on its decision policy 34, a trade off between exploitation of previously chosen workflows and exploration of newly available ones can be decided by the proposed approach.

Definition 5 (Decision Policy). A decision policy $\delta_{t}$, for time $t$, determines a probability distribution of actions over $A(i)$. It is the principle that guides taking action at any state. A policy is a sequence of decision rules. A policy is denoted by $\pi$ and takes the form $\pi=\left(\delta_{1}, \delta_{2}, \ldots, \delta_{t}, \ldots\right)$. Symbol $\pi$ is used for both infinite and finite horizon problems. To obtain a finite horizon policy from an infinite horizon policy, its operation must be restricted to a finite number of time units. A policy tells how to determine actions for any time unit of the process.

To incorporate service composition proactivity, Algorithm 1 is proposed to set the reward values $r$ as follows: for each transaction $t_{j} \in W S_{i}$, the the difference between the aggregated $Q o S$ values $G\left(t_{j}\right)$ (refer to Equation 1) and the mean of the aggregated $Q o S$ values for all transactions $n$ belonging to the same service is computed.

$$
d(j)=G\left(t_{j}\right)-\frac{\sum_{j=1}^{n} G\left(t_{j}\right)}{n}
$$

Then, the sum of $d(j)$ for the last $n$ transactions, referred to as Quality of Expectation $Q o E$, is updated correspondingly.

$$
Q_{o E}=\sum_{j=1}^{n} d(j)
$$

This parameter, $Q o E$ is used as an indicator for possible upward or downward trends in $Q o S$ values of service $W S_{i}$. Low values of $Q o E$ reflect the potential degradation of $Q o S$, while high values of $Q o E$ accommodate for the prospective $Q o E$ growth. When $Q o E$ moves to a negative value, this means that the web service has experienced a series of $Q o S$ degradations and is vulnerable, while positive $Q o E$ values ensure the web service tendency to stability.

The last $n$ transactions are used as a dynamic sliding window to ensure $Q o E$ values keep reflecting latest service activity.

To build proactivity into the proposed approach, $Q o E$ values are used as rewards,

$$
r=Q o E
$$

This empowers the proposed approach to learn the workflow with the least possible potentiality of $Q o S$ degradation, by avoiding degrading workflows and enacting emerging alternatives.

Algorithm 1 presents the proactive adaptation process described above. 


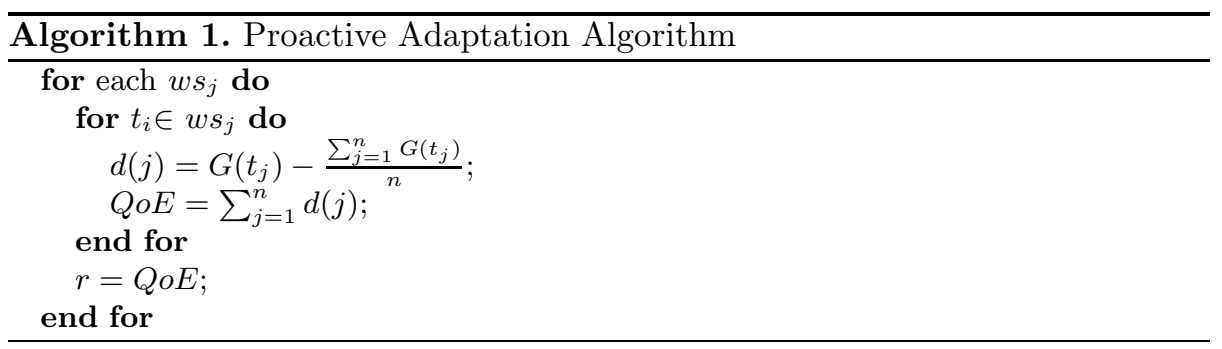

\section{$3 \quad$ Experiment and Analysis}

\subsection{Experiment Set Up}

Two experiments are conducted based on two different considerations. The first experiment explores the optimal learning policy that the proposed approach should follow, whereas the second experiment examines the proactive and adaptive performance of the proposed approach in a highly dynamic web service composition environment. For both experiments, a number of parameters are set up as follows. To simulate a fairly complex dynamic web service composition environment, the number of states $s$, denoting workflows, is set to 3000 and the number of actions $a$, denoting services, is set to 3 per state. To ensure the highest learning efficiency, the learning rate $\alpha$ is set to 1 (refer to Equation 2). The $Q o S$ attributes $Q_{i}$ that will be measured are availability and reliability (refer to Definition 1)and their relative weight $w$ is set to 0.5 each ( refer to Equation 1). This experiment is conducted on $3.33 \mathrm{GHz}$ Intel core 2 Duo PC with $3 \mathrm{~GB}$ of RAM.

\subsection{Result Analysis}

The results of the two experiments are demonstrated and analyzed in details in the following subsections.

Experiment 1. In this experiment, the optimal learning policy is addressed through the discount factor $\gamma$ (refer to Equation 2). To enact an effective learning policy, a trade off between exploration and exploitation should be considered when setting the value of $\gamma$. Low values of $\gamma$ drive that the proposed approach is greedy to exploit the already adopted workflows with immediate rewards, where high values make the proposed approach eager to explore other workflows possibly giving higher expected rewards in the long term. The proposed approach converges to a near optimal policy once it receives the same approximate value of accumulative rewards for a number of successive episodes. Those accumulated rewards are compared episode by episode and the difference is projected against a threshold. For both experiments, this threshold value is set to 0.0001, and the number of successive episodes is set to 1000 . 


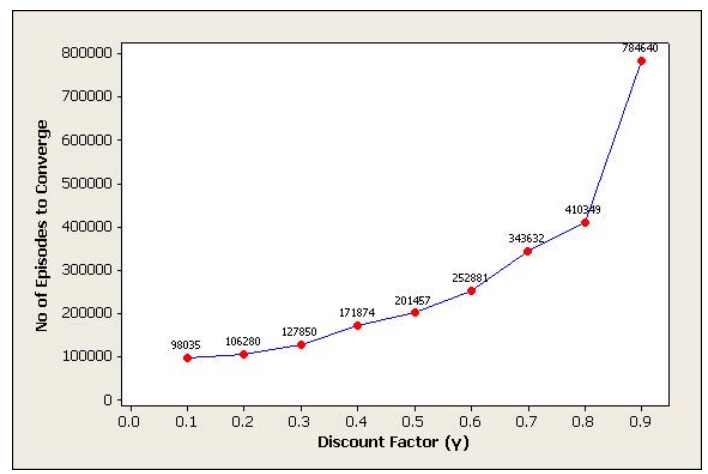

Fig. 1. Learning Policy

Table 2. Learning Policy

\begin{tabular}{|c|c|}
\hline Discount Factor $\gamma$ & No of Episodes to Converge \\
\hline \hline 0.1 & 98035 \\
\hline 0.2 & 106280 \\
\hline 0.3 & 127850 \\
\hline 0.4 & 171874 \\
\hline 0.5 & 201457 \\
\hline 0.6 & 252881 \\
\hline 0.7 & 343632 \\
\hline 0.8 & 410349 \\
\hline 0.9 & 784640 \\
\hline
\end{tabular}

Table 2 shows a scale of $\gamma$ values from 0.1 to 0.9 which are implemented to the proposed approach and the number of episodes till convergence is figured, correspondingly.

Figure 1 depicts the relationship between this group of $\gamma$ values ( $x$ axis) and the corresponding number of episodes needed to converge ( $y$ axis). The trend line shows an upward movement describing a linear relationship, with the number of episodes to converge surges for values of $\gamma \dot{i} 0.8$. This means that more run time and cost incurred in considering longer term rewards. For the rest of the two experiments, the value of $\gamma$ is chosen to be 0.8 as the authors believe that it makes a good trade off between the accumulated rewards gained in the long term and the execution time and cost.

Experiment 2. Experiment 2 tends to reveal how the proposed approach adaptively corresponds to the change in the composition environment. The dynamic change in the composition environment is expressed by the change in the QoS values. Those QoS values may go up or drop down for many reasons. Hence, affecting the reward values $r$ given to the proposed approach (refer to Equations 
$3,4,5)$. The dynamic change in the composition environment is measured in this experiment by two factors. The first factor is the scale of change, that means how many services in the ongoing composition are exposed to QoS changes. The second factor is the frequency of this change, that means how frequently those services realize new QoS values. To recognize the former factor, the QoS values are periodically varied with $1 \%, 5 \%$ and $10 \%$ respectively, every 5000 episodes. Figure 2 depicts the outcome of this change in the composition environment on the performance of the proposed approach. In Figure 2, $x$ axis represents the percentage of $Q o S$ change and $y$ axis represents the convergence time. We can see from Table 3 that it takes 123 and 171 seconds respectively for the proposed approach to converge in a composition environment with $1 \%$ and $5 \%$ periodic scale of change. Compared to 120 seconds in a static composition environment with $0 \%$ scale of change. Those values also show the effectiveness of the proposed approach in learning the optimal policy proactively in a complex composition environment. This convergence time increases slightly to 304 seconds within $10 \%$ scale of change which is rational in such highly adaptive and a fairly complex composition environment.

To experiment the frequency of change, a $5 \%$ scale of change in $Q o S$ is selected and an experiment is conducted to explore the impact of the frequency of change on the time needed by the proposed approach to converge. The frequency of change is represented by the number of episodes the proposed approach executes before receiving new QoS values. Figure 3 depicts this impact with $x$ axis represents the frequency of change and $y$ axis represents the convergence time.

As in Table 4, the proposed approach spends 162 and 171 seconds respectively to converge under frequency of change as 5000 and 10000 episodes respectively. This small difference in time proves the effectiveness of the proposed approach though the frequency of change is doubled from 5000 to 10000 episodes. Finally, the proposed approach takes 230 seconds to converge under a frequency of change every 2500 episodes which is reasonable as in such highly dynamic composition environment.

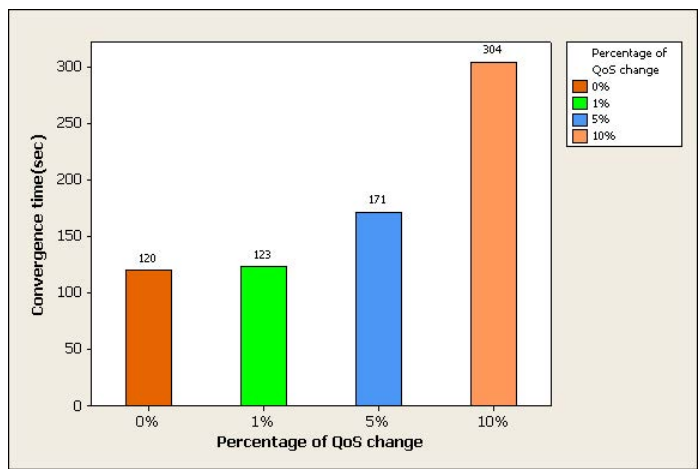

Fig. 2. Scale of Change 
Table 3. Scale of Change

\begin{tabular}{|c|c|}
\hline Percentage of $Q o S$ Change & Convergence Time(sec) \\
\hline \hline $0 \%$ & 120 \\
\hline $1 \%$ & 123 \\
\hline $5 \%$ & 171 \\
\hline $10 \%$ & 304 \\
\hline
\end{tabular}



Fig. 3. Frequency of Change

Table 4. Frequency of Change

\begin{tabular}{|c|c|}
\hline Frequency of Change (episodes) & Convergence Time (sec) \\
\hline \hline 2500 & 162 \\
\hline 5000 & 171 \\
\hline 10000 & 230 \\
\hline
\end{tabular}

\section{Related Work and Discussions}

Various approaches have been proposed in literature to tackle the problem of dynamic composition of web services. In those approaches, two goals have been set for the sake of this purpose. As one point of view, a service composition should provide the functionalities required by service requesters. As another point of view, the quality of a service composition should be maximized. The previous approaches mostly address the two issues in two distinct stages.

In the first stage, a set of tools are used to create an abstract workflow of a service composition, which aims to satisfy users requirements. An example is the work of [9]. They proposed an approach to locate services conforming to independent global constraints. In this approach a greedy algorithm is used to identify conforming values and to locate composite services. Given user constraints, complying values are derived using domain rules. Also, those domain rules are used to combine services assigning those values to their restricted attributes. 
It was observed that the requester and the provider both constrains play a role in composition plan generation involving sequential and parallel execution flows.

Artificial Intelligence (AI) planning methods have also been used for creating abstract workflows of service compositions. In such techniques, both the initial state and the goal state are specified in the requirement by web service requester. AI planning composition techniques employ various methods including: Declarative composition, Situation calculus, Rule based composition, Theorem proving and Graph plan based composition [141812. The search based composition methods are categorized as: forward search based composition and backward search based composition. In goal driven forward search based composition [12], the composition plan is generated in two phases. Firstly, selecting web services that match the requester's input values. Secondly, expanding the plan by matching other services inputs with the outputs of the services identified earlier. In backward search based methods, the composition plan is initialized by recognizing a suitable service that satisfies the requester's outputs. Generally, backward search is more efficient as it does not permit meaningless expansion to the composition plan. An apparent draw back of the previous approaches is that they do not capture all the features of the composition. For example, single web services cannot be directly integrated into the running composition dynamically. In contrast, our learning based approach is able to incorporate both single web services and composite workflows into the undergoing composition automatically at run time.

In the second stage, an optimal set of concrete services are selected to deploy the abstract workflow. This stage is also known as service optimization. UML based modeling techniques have been applied to Aspect Oriented Programming (AOP) technologies for service optimization. In [17], an approach is proposed to address optimization and dynamic adaptation problems. In [13, a decoupling approach based upon the separation of core service logics from context related functionalities was proposed. This separation is conducted via adopting a model driven approach based upon a modified version of the context UML meta model. At the modeling level, core service logics and context handling are treated as independent separate concerns; later AOP encapsulates context dependent behavior.

Few researchers have used the finite state machine (FSM) to model service behavior towards the automatic composition and optimization of web services. In [8], the authors worked on an augmentation based method to model services as FSMs with linear counters to integrate activity processing costs to the model. Automatic e-service optimization is proposed in [2] which presents a framework to describe the exported behavior of an e-service in terms of its possible executions trees. The framework is also specialized to consider exported behavior (i.e. the execution tree of the e-Service) that is represented by a finite state machine. However, as those approaches assume that information about $Q \circ S$ is known and up to date, they cannot deal with the real world cases where such information is unknown. A basic difference between our approach and the previous approaches is that our service composition approach does not depend on 
an static workflow. In real service composition environments, the environment parameters, represented as $Q o S$ values, keep changing in a random and dynamic way. The $Q o S$ values of some services may drop down for many reasons, e.g., the service provider stops functioning. Also, The discovery of new web services with higher $Q_{o S}$ values and getting them involved in the composition workflow to fulfill the requestor's complex requirements. As web services requests are inherently stochastic and web service execution environments are not deterministic, it becomes inappropriate to select and compose web services in a static way. Conversely, service composition in our approach is able to integrate multiple alternative workflows and services. Moreover, our approach does not need to know the non-functional properties of composed services a-priori. Instead, it applies reinforcement learning to derive the optimal solution by analyzing the results of execution. Therefore, our approach of service composition can be highly adaptive to a dynamic environment. Furthermore, by using $Q o E$ values as rewards for Q-learning, our approach is capable to adapt to dynamic changes in the composition environment proactively.

\section{Conclusion}

In this paper, the focus is to incorporate proactive and adaptive behaviors in web service composition under dynamic environments. The proposed approach uses reinforcement learning to proactively adapt to dynamic changes in complex web service composition environments. The sum of the differences of the aggregated QoS values is used as an accumulative reward value. The proposed approach is able to adapt proactively, not only reactively to QoS fluctuations considering both potential degradation and emergence of $Q o S$ values. The results of our experiments show the effectiveness of the proposed approach in highly complex and dynamic web service composition environments.

\section{References}

1. Agarwal, V., Jalote, P.: From specification to adaptation: An integrated qos-driven approach for dynamic adaptation of web service compositions. In: Proc. IEEE Int Web Services (ICWS) Conf., pp. 275-282 (2010)

2. Berardi, D., Calvanese, D., De Giacomo, G., Lenzerini, M., Mecella, M.: Automatic Composition of E-services That Export Their Behavior. In: Orlowska, M.E., Weerawarana, S., Papazoglou, M.P., Yang, J. (eds.) ICSOC 2003. LNCS, vol. 2910, pp. 43-58. Springer, Heidelberg (2003)

3. Chen, G., Cao, W., Chen, X., Wu, M.: Multi-agent q-learning with joint state value approximation. In: Proc. 30th Chinese Control Conf (CCC), pp. 4878-4882 (2011)

4. Clausen, C., Wechsler, H.: Quad-q-learning 11(2), 279-294 (2000)

5. Doshi, P.: Dynamic workflow composition using markov decision processes. International Journal of Web Services Research 2, 1-17 (2005)

6. Gaaloul, K., Walid, Godart, C.: Log-based mining techniques applied to web service composition reengineering. Service Oriented Computing and Applications 2, 93-110 (2008), doi:10.1007/s11761-008-0023-6 
7. Gao, A., Yang, D., Tang, S., Zhang, M.: Web Service Composition Using Markov Decision Processes. In: Fan, W., Wu, Z., Yang, J. (eds.) WAIM 2005. LNCS, vol. 3739, pp. 308-319. Springer, Heidelberg (2005)

8. Gerede, C.E., Ibarra, O.H., Ravikumar, B., Su, J.: Minimum-cost delegation in service composition. Theor. Comput. Sci. 409, 417-431 (2008)

9. Gooneratne, N., Tari, Z.: Matching independent global constraints for composite web services. In: Proceeding of the 17th International Conference on World Wide Web, WWW 2008, pp. 765-774. ACM, New York (2008)

10. Lee, J.W.: Stock price prediction using reinforcement learning. In: Proceedings of IEEE International Symposium on Industrial Electronics, ISIE 2001, vol. 1, pp. 690-695 (2001)

11. Li, H., Dagli, C.H., Enke, D.: Short-term stock market timing prediction under reinforcement learning schemes. In: IEEE International Symposium on Approximate Dynamic Programming and Reinforcement Learning, ADPRL 2007, pp. 233-240 (April 2007)

12. Oh, S.-C., Lee, D., Kumara, S.R.T.: A comparative illustration of ai planning-based web services composition. SIGecom Exch. 5, 1-10 (2006)

13. Prezerakos, G.N., Tselikas, N.D., Cortese, G.: Model-driven composition of contextaware web services using contextuml and aspects. In: IEEE International Conference on Web Services, ICWS 2007, pp. 320-329 (July 2007)

14. Rao, J., Su, X.: A Survey of Automated Web Service Composition Methods. In: Cardoso, J., Sheth, A.P. (eds.) SWSWPC 2004. LNCS, vol. 3387, pp. 43-54. Springer, Heidelberg (2005)

15. Tang, R., Zou, Y.: An approach for mining web service composition patterns from execution logs. In: Proc. IEEE Int Web Services (ICWS) Conf., pp. 678-679 (2010)

16. Watkins, C.: Learning from Delayed Rewards. PhD thesis, Cambridge University, England (1989)

17. Xu, Y., Tang, S., Xu, Y., Tang, Z.: Towards aspect oriented web service composition with uml. In: 6th IEEE/ACIS International Conference on Computer and Information Science, ICIS 2007, pp. 279-284 (July 2007)

18. Zahoor, E., Perrin, O., Godart, C.: Rule-based semi automatic web services composition. In: Proceedings of the 2009 Congress on Services - I, pp. 805-812. IEEE Computer Society, Washington, DC (2009) 